\title{
EPIDEMIOLOGICAL STUDY ON MORE ACCURATE DIAGNOSIS OF PROSTATE CANCER
}

\author{
Josef Kopecký ${ }^{1}$, Veronika Navláčilová ${ }^{2}$, Jana Janoutová ${ }^{3}$, Vladimír Janout ${ }^{3}$ \\ 'Urology Department, Haviřov Hospital, Haviř̃ov, Czech Republic \\ ${ }^{2}$ Medihope Ltd., Olomouc, Czech Republic \\ ${ }^{3}$ Faculty of Health Sciences, Palacký University Olomouc, Olomouc, Czech Republic
}

\begin{abstract}
SUMMARY
Objective: The study aimed at assessing the potential benefit of prostate health index (PHI) for early detection of prostate cancer (PCa) and the use of $\mathrm{PHI}$ as a marker predicting the presence of $\mathrm{PC}$ a before performing prostate biopsy.

Methods: The study comprised 55 males who underwent prostate biopsy. Before the procedure, blood samples were collected to test prostate specific antigen (PSA) and free/total PSA ratio (\% FPSA) and PHI was calculated. Receiver operating characteristic (ROC) analysis was used to assess the benefit of these values for predicting the presence of $\mathrm{PCa}$.

Results: Based on histological examination 31 males were diagnosed with PCa, the remaining 24 were negative. Among the PCa patients, $39 \%$ had a Gleason score of $6,26 \%$ had a score of 7 and $35 \%$ had a score of $8-10$. There were statistically significant differences in PHI and PSA between males with and without PCa. The areas under the ROC curve for \%fPSA, total PSA and PHI were $0.712,0.746$ and 0.789 , respectively. $\mathrm{PHI}$ showed the best predictive ability to estimate biopsy results. If the cut-off criterion $\mathrm{PHI}>36.4(77.42 \%$ sensitivity, $66.67 \%$ specificity) had been used, $41.7 \%$ of males would have avoided unnecessary biopsy.

Conclusion: The use of PHI may considerably improve the accuracy of $\mathrm{PC}$ a detection in patients with elevated $\mathrm{PSA}$ and thus reduce the number of unnecessary biopsies.
\end{abstract}

Key words: prostate cancer, prostate specific antigen, prostate health index, biopsy

Address for correspondence: V. Janout, Faculty of Health Sciences, Palacký University Olomouc, Hněvotínská 976/3, 77515 Olomouc, Czech Republic. E-mail: vladimir.janout@upol.cz

https://doi.org/10.21101/cejph.a5720

\section{INTRODUCTION}

Prostate cancer $(\mathrm{PCa})$ is currently the most frequent malignancy in males (1). The incidence of PCa has increased four-fold over the last decades and this steep increase is attributed to the introduction of the prostate specific antigen (PSA) test into clinical practice. Early detection and subsequent effective treatment of $\mathrm{PCa}$ are important for prolonging patient lives. A prostate biopsy plays a key role in confirming the diagnosis and early initiation of therapy. With the prostate biopsy, PCa may be histologically confirmed. Before the biopsy is performed, its benefit for the particular patient must be considered. The main indications for performing the biopsy are a suspicious digital rectal examination (DRE) or other abnormal parameters assessing the risk of $\mathrm{PCa}$ such as PSA or prostate health index (PHI).

PSA is a glycoprotein produced by prostatic acinar and ductal epithelial cells. The antigen is an enzyme; its primary function is to liquefy human semen. It is an organ-specific marker. Under normal circumstances, its level is low; it is measured in $\mathrm{ng} / \mathrm{mL}$. The cut-off for performing a biopsy is $3.5 \mathrm{ng} / \mathrm{mL}^{*}$. The amount of PSA is strongly influenced by androgen production. Increased
PSA may be contributed to by multiple factors, for instance impaired cell integrity in prostate diseases such as infection or benign prostatic hyperplasia and mechanical manipulation of the prostate during examination. Thus, elevated PSA does not necessarily mean the presence of PCa. In blood serum, PSA is in a free (fPSA) and bound (tPSA) forms, at a ratio of $1: 4(2,3)$. The PSA value itself has a very low specificity with a positive predictive value of approximately $25 \%$, leading to high rates of false-positive results and $75 \%$ of unnecessary prostate biopsies (4).

In clinical practice, the PSA test is easily available and, when combined with DRE, it has improved PCa detection. Although the serum PSA cut-off has been set at $4 \mu \mathrm{g} / \mathrm{L}$, as many as $20 \%$ of PCa patients have lower levels. With regard to cancer detection, the PSA sensitivity and specificity are reported to be $68-80 \%$ and $49-90 \%$, respectively. In case of benign prostatic disease, serum PSA is usually higher. The f/t PSA ratio may be considered useful particularly with total PSA ranging from 4 to $10 \mathrm{ng} / \mathrm{mL}$; the predictive value decreases with lower values $(5,6)$.

ProPSA is produced by prostate cells as a precursor of PSA and gradually transformed to the definitive form of PSA by enzymatic processes. It exists in several isoforms in blood and its

\footnotetext{
*https//uroweb.org/guideline/prostate-cancer/
} 
normal level accounts for approximately $30 \%$ of the total fPSA amount. ProPSA is very sensitive and it aids in decision-making about performing a biopsy if PSA is increased. The [-2]proPSA isoform (also written as p2PSA) is useful in case of a suspicious DRE and elevated PSA as it increases clinical significance in cancer detection. Compared to tPSA and f/tPSA, its specificity is higher. With immunohistochemistry staining, its activity is higher in tumor than benign tissue. This value is used to determine PHI and $\%$ p2PSA calculated as p2PSA/fPSA (7).

In recent years, research in urology has focused on novel serum biomarkers potentially improving PCa diagnosis. PSI, a novel promising test based on PSA, has been the subject of many studies. Since approximately $75 \%$ of prostate biopsies are performed unnecessarily, it is important to find a test that is as specific as possible to reduce unnecessary biopsies. This is what PHI offers (8). PHI is a value determined by a mathematical calculation combining tPSA, fPSA and the [-2]proPSA isoform. The index is calculated using the following formula: ([-2]proPSA/freePSA)

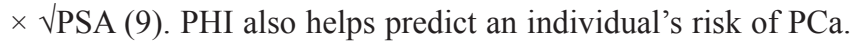
The highest predictive value is when tPSA ranges between 2 and $10 \mathrm{ng} / \mathrm{mL}(10)$. The introduction of this marker into PCa diagnosis reduces the potential risk of unnecessary biopsies and subsequent wrong diagnosis and treatment. Studies on errors in the diagnosis of $\mathrm{PCa}$ have produced varied results. A study by Welch and Albertsen reported the overdiagnosis of 1.3 million males in the USA in 1987-2005 (11). Given its high accuracy in predicting $\mathrm{PCa}$, $\mathrm{PHI}$ value based on calculation using p2PSA might considerably contribute to a lower rate of negative prostate biopsies, reducing the costs of diagnosis and therapy (12). Moreover, prostate biopsy puts a lot of mental stress on the patient and there is a risk of consequences (hemorrhagic complications, potency problems and infection risk).

The study aimed at assessing the benefit of PHI for PCa diagnosis and the use of $\mathrm{PHI}$ as a marker in the diagnostic process when making decision about performing prostate biopsy. The key assessments were sensitivity, specificity and diagnostic accuracy of PHI (as a test) for predicting the presence of PCa as compared with tPSA and \%fPSA (standard tests).

\section{MATERIALS AND METHODS}

Patients suspected of having PCa were examined at the Urology Department of Havírov Hospital between January and June 2015. Before biopsies were performed, blood was collected from all participants to determine PSA and its derivatives key for subsequent assessment of relationships between these values and the presence of PCa. During the biopsy procedure tissue samples were obtained from both prostate lobes. In more than half of patients, sextant biopsies were performed, with 6 tissue samples taken from each lobe, that is, a total of $6+6$ samples. Biopsy indication criteria were not limited by age, level of PSA or other, because the aim of the study was not to find new cases of PCa. Biopsy indication criteria was established by attending urologist (high level of PSA or progression of PSA with respect to size of prostate gland). All males who volunteered to participate gave written informed consent to anonymous data analysis in the study. Basic descriptive statistics were used to characterize the participants (median, arithmetic mean, minimum, maximum and standard deviation - SD). Differences in values between the groups with and without PCa were analyzed with an independent $t$-test assessed at a level of significance of 5\%. Data were statistically analyzed using MedCalc (Version_12.4.0.0*). For individual values of the sample, total PSA, \%PSA and PHI, receiver operating characteristic (ROC) analysis was performed (test sensitivity, specificity, area under the curve - AUC, SD, confidence interval).

ROC analysis is an instrument for the assessment and optimization of a classification system (test) that shows a relationship between specificity and sensitivity of the test for all possible threshold values. The higher the specificity and sensitivity are, the more useful the test (diagnostic method) is. In an ROC graph, the best diagnostic test has the largest AUC. In the literature, AUC classifications quantifying the diagnostic power of a test may be found. According to the most frequently used classification, a test with an AUC greater than 0.75 may be considered able to provide satisfactory discrimination (13).

\section{RESULTS}

The study comprised 55 male patients suspected of having PCa. Prior to biopsy, blood samples were analyzed in the laboratory. All participants underwent a prostate biopsy for elevated PSA. Based on histological examination of prostate tissue biopsy samples, PCa was confirmed in 31 males. In the remaining 24 males, the biopsy yielded negative results. In patients with both positive and negative findings, the distribution of PHI was as follows: a total of 15 males ( 3 positive, 12 negative) in the $\mathrm{PHI} \leq 28$ category (according to Beckman Coulter); a total of 6 males (4 positive, 2 negative) in the PHI 29-36 category; a total of 12 males (6 positive, 6 negative) in the PHI 37-54 category; and a total of 22 males (18 positive, 4 negative) in the $\mathrm{PHI} \geq 55$ category. In males with positive $\mathrm{PCa}$ findings, $\mathrm{PHI} \geq 55$ prevailed; in those with negative results, by contrast, $\mathrm{PHI} \leq 28$ was most common (Fig. 1).

Males with positive results had a median PSA of $12.8 \mathrm{ng} / \mathrm{L}$ as compared with $5.94 \mathrm{ng} / \mathrm{L}$ in those with negative results; their median prostate tissue volumes were $36 \mathrm{~mL}$ and $39 \mathrm{~mL}$, respectively. There was a clear difference in the median PHI between males with positive (59.40) and negative (30.85) findings (Table 1). Among 31 patients diagnosed with PCa, $12(39 \%)$ had a Gleason score of $6,8(26 \%)$ had a score of 7 and the remaining $11(35 \%)$ had a score of 8 or more (Fig. 2).

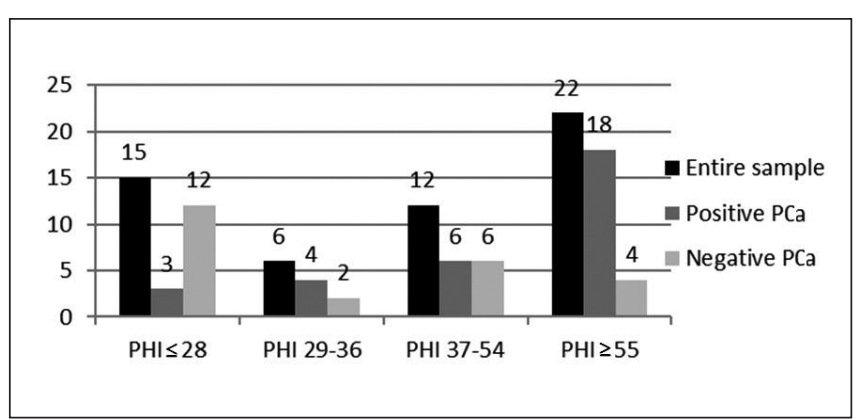

Fig. 1. Distribution of $\mathrm{PHI}$ in individual subgroups. 
Table 1. Clinical characteristics of the patient sample $(N=55)$

\begin{tabular}{|c|c|c|c|}
\hline & $\begin{array}{c}\text { Positive finding } \\
n=31\end{array}$ & $\begin{array}{l}\text { Negative finding } \\
\qquad n=24\end{array}$ & $\mathrm{p}$-value \\
\hline PSA - median & 12.80 & 5.94 & \\
\hline Arithmetic mean & 24.3 & 7.5 & $<0.001$ \\
\hline Min-max (ng/mL) & $3.12-150.00$ & $1.17-18.50$ & \\
\hline PHI - median & 59.40 & 30.85 & \\
\hline Min-max & $20.5-456.0$ & $14.0-148.0$ & \\
\hline Arithmetic mean & 94.4 & 38.4 & $<0.001$ \\
\hline$\%$ fPSA - median & 10.01 & 15.34 & \\
\hline Arithmetic mean & 12.7 & 17.2 & 0.734 \\
\hline Min-max & $4.65-29.56$ & $7.14-32.00$ & \\
\hline Volume - median & 36 & 39 & 0.038 \\
\hline Min-max (mL) & $18.7-80.0$ & $16.0-90.0$ & \\
\hline \multicolumn{4}{|c|}{ Gleason score, n (\%) } \\
\hline GS 6 & $12(38.71)$ & & \\
\hline GS 7 & $8(25.81)$ & & \\
\hline GS 8-10 & $11(35.48)$ & & \\
\hline
\end{tabular}

ROC analysis showed the best diagnostic accuracy for predicting the presence of PCa in PHI. The AUC was 0.789, p $<0.001$ (95\% CI: 0.658-0.887). Each possible value of PHI in the sample was assigned test specificity and sensitivity. The best compromise between sensitivity and specificity was a cut-off criterion for early $\mathrm{PCa}$ detection of $\mathrm{PHI}>36.4$ with $77.42 \%$ sensitivity and $66.67 \%$ specificity of the test (Fig. 3 ). If this cut-off criterion had been used in the sample, 23 males (41.8\%) would have avoided a prostate biopsy and there would have been 7 false-negative cases.

Just the second, after PHI, was the PSA test, with an AUC of $0.746, \mathrm{p}<0.001$ (95\% CI: 0.611-0.854). The best cut-off discriminating between positive and negative findings was PSA $>9.57$ ng/mL with $67.74 \%$ sensitivity and $75 \%$ specificity (Fig. 4). Had this cut-off criterion been used in the sample, 28 males (50.9\%) would have avoided an unnecessary prostate biopsy; however, the number of false-negative cases would have been 10 .

For the \%fPSA test, the AUC was 0.712, $\mathrm{p}=0.0027(95 \% \mathrm{CI}$ : $0.573-0.850)$. The best cut-off discriminating between positive and negative findings was $\%$ fPSA $<11.22$ with $58.06 \%$ sensitivity and $91.67 \%$ specificity (Fig. 5). With this cut-off criterion, 32 males $(58.2 \%)$ would have avoided a prostate biopsy but 14 of them would have been false-negatively diagnosed.

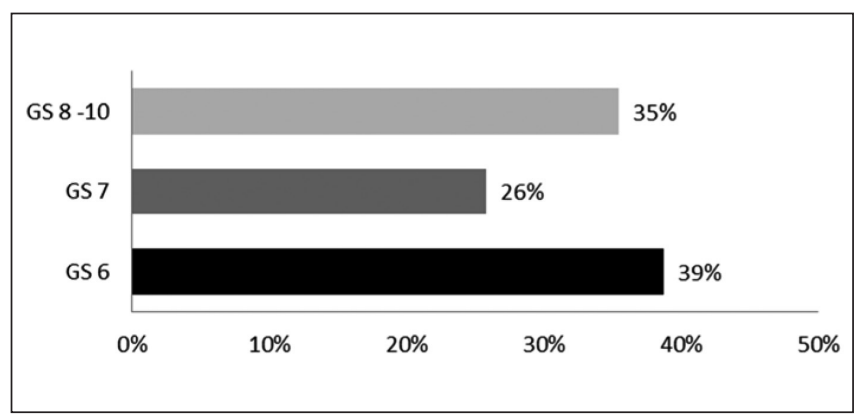

Fig. 2. Distribution of Gleason scores in patients with prostate cancer.

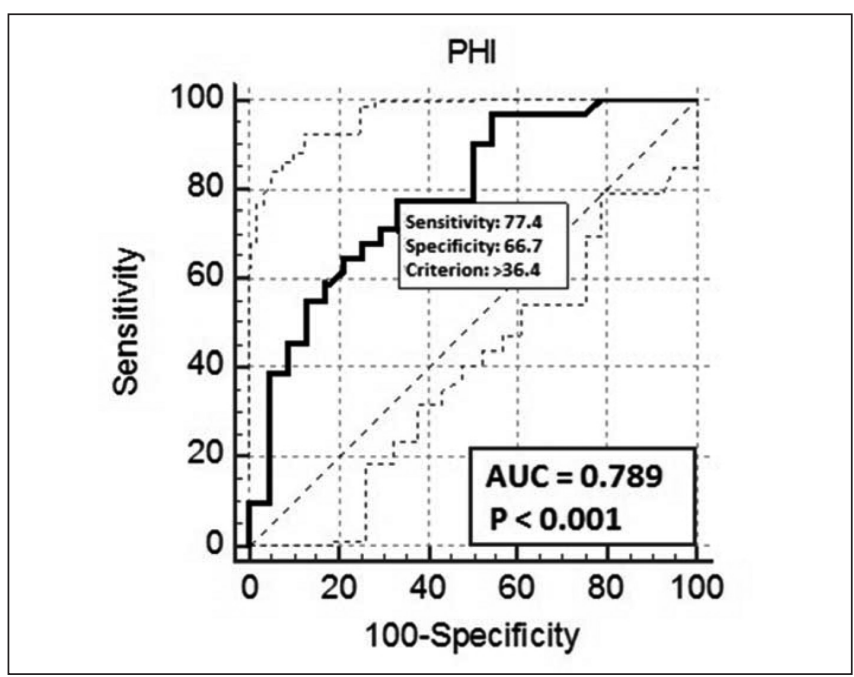

Fig. 3. Area under the curve (AUC) - PHI.

The ROC analysis is summarized in Table 2. Comparison of $\%$ fPSA, tPSA and PHI values suggests that the PHI test showed the best diagnostic accuracy for predicting $\mathrm{PCa}$ and thus potentially the greatest impact on reducing the number of unnecessary biopsies. When considering the need for a biopsy, PHI together with PSA seem to be very beneficial as compared with PSA alone.

For patients, ROC analysis was performed using all values in the sample. For comparison, analysis was conducted for males with PSA ranging from 1 to $10 \mathrm{ng} / \mathrm{mL}$. The results are summarized in Table 3. For the entire sample, the largest AUC was seen in PHI (AUC 0.789, p <0.001), followed by PSA (AUC 0.746, p <0.001) and $\%$ fPSA (AUC 0.712, $\mathrm{p}=0.003$ ). For males with PSA below $10 \mathrm{ng} / \mathrm{mL}(\mathrm{n}=28)$, the largest AUC was seen in PHI (AUC = $0.711, p=0.037)$, followed by \%fPSA $(A U C=0.650, p=0.196)$, and PSA $(\mathrm{AUC}=0.567, \mathrm{p}=0.564)$. In both subgroups, PHI was superior to both PSA and \%fPSA, suggesting that it carries the greatest weight when considering a biopsy. 


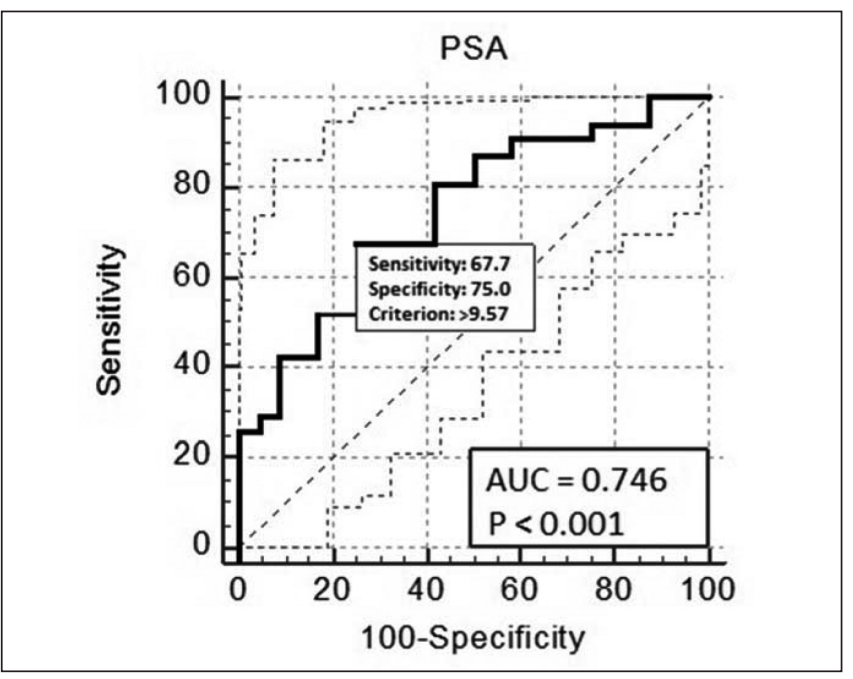

Fig. 4. Area under the curve (AUC) - PSA.

\section{DISCUSSION}

The most frequent incentive for considering the presence of PCa is an increased PSA level. However, this may be frequently confounded by many factors such as age, benign prostatic hyperplasia or prostatitis. Elevated PSA levels are associated with a higher risk for PCa, namely $25 \%$ for PSA $4 \mathrm{ng} / \mathrm{mL}$ and as much as $50 \%$ for PSA $>10 \mathrm{ng} / \mathrm{mL}$ (14).

Vukotic et al. (15) reported the risk of the presence of $\mathrm{PCa}$ of $20 \%$ for patients with PSA $4-10 \mathrm{ng} / \mathrm{mL}$ and as much as $33 \%$ for those with PSA $10-20 \mathrm{ng} / \mathrm{mL}$. In the present study, $49 \%$ of males had PSA $>10 \mathrm{ng} / \mathrm{mL}$ which is consistent with the above facts. Based on biopsy results, 31 males were diagnosed with

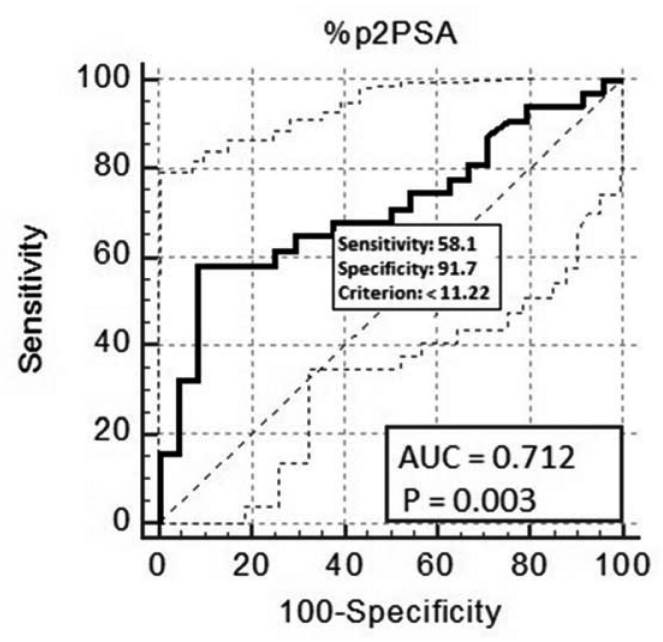

Fig. 5. Area under the curve (AUC) - \%fPSA.

$\mathrm{PCa}$, that is over half of the entire sample ( 55 males). Histological examination showed GS 6 in 39\%, GS 7 in 26\% and GS $8-10$ in $35 \%$ of males. According to the Institute of Health Information and Statistics of the Czech Republic, the number of early detected cases with low-grade PCa has dramatically increased since 2012. The increase is attributed to introduction of routine PSA tests into clinical practice and novel diagnostic tests.

Recently, studies have focused on novel, clinically more important markers shown to provide better diagnostic yield such as fPSA, \%fPSA and PHI. Theoretically, there are 7 proPSA isoforms, with p2PSA being clinically the most significant. This marker is used for calculating PHI. A considerable benefit of p2PSA has been reported by authors of numerous studies. In their

Table 2. Summary of ROC analysis

\begin{tabular}{|l|c|c|c|c|c|c|c|}
\hline & $\begin{array}{c}\text { Area under } \\
\text { ROC curve }\end{array}$ & SE $^{\mathrm{a}}$ & $\mathbf{9 5 \%} \mathbf{C l}^{\mathrm{b}}$ & p-value & Cut-off criterion & Sensitivity & Specificity \\
\hline PHI & 0.789 & 0.0621 & $0.658-0.887$ & $<0.001$ & $>36.40$ & $77.42 \%$ & $66.67 \%$ \\
\hline tPSA & 0.746 & 0.0665 & $0.611-0.854$ & $<0.001$ & $>9.57$ & $67.74 \%$ & $75.00 \%$ \\
\hline$\%$ fPSA & 0.712 & 0.0706 & $0.573-0.850$ & 0.003 & $<11.22$ & $58.06 \%$ & $91.67 \%$ \\
\hline
\end{tabular}

aDeLong et al., 1988 (18)

${ }^{\mathrm{b}} \mathrm{AUC} \pm 1.96 \mathrm{SE}$

Table 3. Analysis of ROC curves for individual values

\begin{tabular}{|l|l|c|}
\hline \multirow{2}{*}{$\begin{array}{l}\text { Entire sample (all PSA values) } \\
n=55\end{array}$} & PCa - AUC (95\% Cl) & p-value \\
\hline \%fPSA & Positive findings: 31 & 0.003 \\
\cline { 2 - 3 } & Negative findings: 24 & $<0.001$ \\
\hline PSA & $0.712(0.537-0.850)$ & $<0.001$ \\
\hline PHI & $0.746(0.611-0.854)$ & \\
\hline Sample with PSA 1-10 ng/mL & $0.789(0.658-0.887)$ & \\
\hline \%fPSA & Positive findings: 10 & 0.196 \\
\hline PSA & Negative findings: 18 & 0.564 \\
\hline PHI & $0.650(0.448-0.819)$ & 0.037 \\
\hline
\end{tabular}


study of 157 males with normal DRE findings undergoing their first prostate biopsy, Tan et al. analyzed various markers including p2PSA. They found a statistically significant difference in p2PSA between males with and without cancer $(p=0.001)$. ROC analysis of p2PSA determined the AUC at 0.695 (95\% CI: 0.592-0.797), $\mathrm{p}=0.004$, indicating a discriminating test (16).

The present study assessed PSA, \%fPSA, prostate volume and PHI. There were statistically very significant differences in PSA and PHI ( $p>0.001)$ between males with and without PCa.

The best diagnostic accuracy for predicting cancer was observed in PHI. ROC analysis showed AUC $=0.789$ (95\% CI: $0.658-0.887), p>0.001$. This was the largest AUC in the sample, clearly confirming the expected fact that PHI had the best diagnostic effect in these patients. The cut-off criterion was found to be PHI $>36.4$ with $77.42 \%$ sensitivity and $66.67 \%$ specificity. Had this cut-off criterion been used in the sample, 23 males (41.8\%) would have avoided an unnecessary prostate biopsy. Available studies assessing the role of various PSA derivatives in predicting the presence of PCa showed superiority of PHI and its AUC over the others. In a recent study of 154 males with PSA between 4 and $10 \mathrm{ng} / \mathrm{mL}$ undergoing biopsy, PCa was diagnosed in 36 patients. There were statistically significant differences in \%fPSA, \%p2PSA and PHI between males with and without PCa. The best diagnostic test was PHI, with an AUC of 0.77. The best cut-off criterion was $\mathrm{PHI}>29$.6. If this cut-off criterion had been used in the sample, 88 males would have avoided an unnecessary prostate biopsy and there would have been 8 false-negative cases (17).

\section{CONCLUSION}

The study aimed to assess the benefit of PHI for diagnosing $\mathrm{PCa}$ and its use as a marker of this cancer when considering a prostate biopsy. In a sample comprising 55 males, \%fPSA, PSA and PHI were determined and prostate tissue biopsy was performed. Cancer was confirmed in 31 patients. There were statistically significant differences in PSA and PHI between males with and without $\mathrm{PCa}$. ROC analysis showed the best diagnostic accuracy for predicting the presence of PCa in PHI. Compared to the others, PHI had the largest AUC, which made it superior to $\%$ fPSA and PSA. Consistently with authors of other studies, the test had higher sensitivity and specificity. If the cut-off criterion PHI $>36.4$ with $77.42 \%$ sensitivity and $66.67 \%$ specificity had been used, nearly half of the patients would have avoided an unnecessary prostate biopsy; this would have reduced costs spent on this procedure by health insurance companies.

\section{Conflict of Interests}

None declared

\section{REFERENCES}

1. Epidemiology of malignant tumors in the Czech Republic [Internet]. Masaryk University [cited 2019 Oct 17]. Available from: http://www. svod.cz.

2. Král M, Vyhnálková V, Študent V, Bouchal J. Genetic risk of prostate cancer. Ces Urol. 2010;14(3):139-47. (In Czech.)

3. International Agency for Research on Cancer. Global Cancer Observatory [Internet]. [cited 2018 Apr 16]. Available from: https://gco.iarc.fr/.

4. Vukovic I, Djordjevic D, Bojanic N, Babic U, Soldatovic I. Predictive value of [-2]propsa (p2psa) and its derivatives for the prostate cancer detection in the 2.0 to $10.0 \mathrm{ng} / \mathrm{mL}$ PSA range. Int Braz J Urol. 2017 JanFeb;43(1):48-56.

5. Wasserbauer R. Active approach to prostate cancer detection - what is reasonable and what may cause harm. Urol List. 2014;12(2):19-28. (In Czech.)

6. Štern P, Vranovský K, Šafarčík K. Prostatic cancer - molecular basis, diagnostics and economy of prevention. Klin Biochem Metab. 2008;16(37):19-26. (In Czech.)

7. Huang YQ, Sun T, Zhong WD, Wu CL. Clinical performance of serum [-2]proPSA derivatives, \%p2PSA and PHI, in the detection and management of prostate cancer. Am J Clin Exp Urol. 2014 Dec 25;2(4):343-50.

8. Beckman Coulter. Prostate Health Index (phi) [Internet]. Beckman Coulter [cited 2018 Apr 24]. Available from: https://www.beckmancoulter.com/ products/immunoassay/phi.

9. Loeb S, Catalona WJ. The Prostate Health Index: a new test for the detection of prostate cancer. Ther Adv Urol. 2014 Apr;6(2):74-7.

10. Na R, Ye D, Qi J, Liu F, Helfand BT, Brendler CB, et al. Prostate health index significantly reduced unnecessary prostate biopsies in patients with PSA $2-10 \mathrm{ng} / \mathrm{mL}$ and PSA $>10 \mathrm{ng} / \mathrm{mL}$ : results from a Multicenter Study in China. Prostate. 2017 Aug;77(11):1221-9.

11. Welch HG, Albertsen PC. Prostate cancer diagnosis and treatment after the introduction of prostate-specific antigen screening: 1986-2005. J Natl Cancer Inst. 2009 Oct 7;101(19):1325-9.

12. Nichol MB, Wu J, An JJ, Huang J, Denham D, Frencher S, et al. Budget impact analysis of a new prostate cancer risk index for prostate cancer detection. Prostate Cancer Prostatic Dis. 2011 Sep;14(3):253-61.

13. Nichol MB, Wu J, Huang J, Denham D, Frencher SK, Jacobsen SJ. Costeffectiveness of Prostate Health Index for prostate cancer detection. BJU Int. 2012 Aug; 110(3):353-62.

14. Thompson IM, Pauler DK, Goodman PJ, Tangen CM, Lucia MS, Parnes $\mathrm{HL}$, et al. Prevalence of prostate cancer among men with a prostatespecific antigen level $<$ or $=4.0$ ng per milliliter. N Engl J Med. 2004 May 27;350(22):2239-46.

15. Vukotic V, Cerovic S, Kozomara M, Lazic M. The predictive value of PSA in diagnosis of prostate cancer in non screened population. Acta Chir Iugosl. 2005;52(4):81-7.

16. Tan LG, Tan YK, Tai BC, Tan KM, Gauhar V, Tiong HY, et al. Prospective validation of \%p2PSA and the Prostate Health Index, in prostate cancer detection in initial prostate biopsies of Asian men, with total PSA 4-10 ng ml-1. Asian J Androl. 2017 May-Jun;19(3):286-90.

17. Hsieh PF, Chang CH, Yang CR, Huang CP, Chen WC, Yeh CC, et al. Prostate Health Index (PHI) improves prostate cancer detection at initial biopsy in Taiwanese men with PSA 4-10 ng/mL. Kaohsiung J Med Sci. 2018 Aug;34(8):461-6.

18. DeLong ER, DeLong DM, Clarke-Pearson DL. Comparing the areas under two or more correlated receiver operating characteristic curves: a nonparametric approach. Biometrics. 1988 Sep;44(3):837-45.

Received February 20, 2019 Accepted in revised form October 17, 2019 